\title{
Bei hohen Acetylcholin-Rezeptor-Spiegeln besteht Neigung zur Generalisierung
}

Fragestellung: Die Autoren wollten prüfen, ob die Bestimmung von Acetylcholin-Rezeptor-Antikörper-Titern bei Patienten mit okulärer Mysthenia gravis eher, als dies früher vermutet wurde, zu einer Prädiktion bezüglich einer möglichen späteren Generalisierung führen kann.

Hintergrund: Etwa $90 \%$ aller Patienten mit einer Myasthenia gravis weisen Störungen der Augenmuskulatur (Ptose und Diplopie) auf. Etwa die Hälfte der Patienten mit Augenmuskelstörungen hat eine isolierte okuläre Myasthenie. Die Differenzialdiagnose ist mitunter nicht einfach, da Patienten auch eine internukleäre Ophthalmoplegie, eine Schilddrüsenerkrankung oder eine Schädigung der Hirnnerven aufweisen können. Manche der Patienten sprechen auch auf eine immunsuppressive Therapie und insbesondere auf Pyridostigmin nicht an. Somit müssen zusätzliche Tests wie die Bestimmung von Acetylcholin-Rezeptor-Antikörpern im Serum durchgeführt werden. Im Vergleich zur generalisierten Myasthenie, wo mindestens 85-90\% aller Patienten positive Acetylcholin-Rezeptor-Antikörper im Serum aufweisen, ist dies bei Patienten mit okulärer Myasthenie nur bei $50 \%$ der Patienten der Fall. Die Autoren wollten deswegen prüfen, ob Patienten mit positiven Acetylcholin-Rezeptor-Antikörpern eher generalisieren als solche ohne diesen Antikörpernachweis.

Peeler CE, De Lott LB, Nagia L et al. Clinical Utility of Acetylcholine Receptor Antibody Testing in Ocular Myasthenia Gravis. JAMA Neurol 2015; 72 : $1170-4$
Patienten und Methodik: Dazu wurde in der größten mit dieser Fragestellung bisher durchgeführten Studie eine retrospektive Analyse von 223 Patienten mit einem mittleren Alter von 59 Jahren
(62 \% Männer), die zwischen Juli 1986 und Mai 2013 an der Harvard Medical School oder an der Michigan State University diagnostiziert wurden, bezüglich einer späteren Generalisierung untersucht. Dazu wurde das Alter, Geschlecht, die Symptome, die Acetylcholin-Rezeptor-Antikörper-Titer sowie die Progression bis zur Generalisierung für jeden einzelnen Patienten untersucht und statistisch ein Zusammenhang zwischen den klinischen Variablen und den Antikörperergebnissen erstellt. Mittels Kaplan-Meier-Analyse wurde der Zeitrahmen bis zur Generalisierung bestimmt.

Ergebnisse: Unter den 223 Teilnehmern dieser Studie wiesen 158 Patienten (70,9\%) einen positiven Acetylcholin-RezeptorAntikörper-Titer auf. Zur Zeit der Diagnose waren die Patienten im Schnitt 59 Jahre alt, $11 \%$ hatten eine Ptose, 34 \% Doppelsehen und $55 \%$ die Kombination aus Ptose und Doppelsehen. 54 Patienten entwickelten eine generalisierte Myasthenie. Dies entspricht $20 \%$ des untersuchten Kollektivs mit einer Followup-Zeit mit bis zu 60 Monaten. Die Patienten mit positivem Acetylcholin-Rezeptor-Antikörper-Titer waren signifikant älter, als die mit einem negativen Test (61,1 gegenüber 54,7 Jahre). Frauen wiesen deutlich seltener positive Acetylcholin-Rezeptor-Antikörper auf. Die Patienten, die eine Generalisierung entwickelten, hatten deutlich höhere Acetylcholin-Rezeptor-AntikörperTiter, als diejenigen, die später nicht generalisierten.

Schlussfolgerungen: Diese Arbeit zeigt somit, dass deutlich mehr Patienten mit einer okulären Myasthenie als früher angenommen Acetylcholin-Rezeptor-Antikörper positiv sind. Insbesondere ältere und Männer mit hohen Acetylcholin-Rezeptor-Antikörpern neigen zur Progression zu einer generalisierten Myasthenia gravis.

\section{- Kommentar von Heinz Reichmann, Dresden}

\section{Antworten auf eine vieldiskutierte Frage}

Diese Studie bietet sehr hilfreiche Informationen für die stets zu diskutierende Frage, ob Patienten mit einer okulären Myasthenie generalisieren. Aus dieser Studie ist zu lernen, dass es insbesondere bei männlichen und älteren Patienten mit okulären Symptomen sehr wohl zur Generalisierung kommen kann, wenn diese hohe Acetylcholin-Rezeptor-Antikörper aufweisen. Aus meiner Sicht etwas einschränkend ist die Tatsache, dass diese Patienten in hoch spezialisierten Zentren meist zur Bestätigung einer Verdachtsdiagnose gesehen wurden, so dass wir es hier mit einem doch präselektierten Patientenkollektiv zu tun haben. Trotzdem ist es die größte bisher durchgeführte Studie zur Frage, wie viele Patienten mit einer okulären Myasthenie generalisieren.

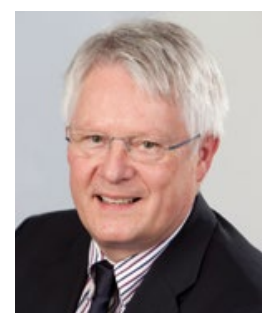

Prof. Dr. med. Heinz Reichmann, Dresden

Direktor der Klinik und Poliklinik für Neurologie, Klinikum Carl Gustav Carus der TU Dresden, Dekan der Medizinischen Fakultät. E-Mail: heinz.reichmann@ uniklinikum-dresden.de 